Draft VERSION OCtOBER 1, 2018

Preprint typeset using $\mathrm{LAT}_{\mathrm{E}} \mathrm{X}$ style emulateapj v. 11/10/09

\title{
X-RAY SPECTROSCOPY OF THE HIGH MASS X-RAY BINARY PULSAR CENTAURUS X-3 OVER ITS BINARY ORBIT
}

\author{
Sachindra NAIK ${ }^{1}$, Biswajit Paul ${ }^{2}$, Zulfikar Ali ${ }^{1}$ \\ Draft version October 1, 2018
}

\begin{abstract}
We present a comprehensive spectral analysis of the high mass X-ray binary (HMXB) pulsar Centaurus X-3 with the Suzaku observatory covering nearly one orbital period. The light curve shows the presence of extended dips which are rarely seen in HMXBs. These dips are seen up to as high as $\sim 40$ $\mathrm{keV}$. The pulsar spectra during the eclipse, out-of-eclipse, and dips are found to be well described by a partial covering power-law model with high energy cut-off and three Gaussian functions for $6.4 \mathrm{keV}$, $6.7 \mathrm{keV}$, and $6.97 \mathrm{keV}$ iron emission lines. The dips in the light curve can be explained by the presence of an additional absorption component with high column density and covering fraction, the values of which are not significant during the rest of the orbital phases. The iron line parameters during the dips and eclipse are significantly different compared to those during the rest of the observation. During the dips, the iron line intensities are found to be lesser by a factor of 2-3 with significant increase in the line equivalent widths. However, the continuum flux at the corresponding orbital phase is estimated to be lesser by more than an order of magnitude. Similarities in the changes in the iron line flux and equivalent widths during the dips and eclipse segments suggests the dipping activity in Cen X-3 is caused by obscuration of the neutron star by dense matter, probably structures in the outer region of the accretion disk, as in case of dipping low mass X-ray binaries.
\end{abstract}

Subject headings: stars: neutron, pulsars: individual: Cen X-3, X-rays: stars

\section{INTRODUCTION}

Orbital phase dependent dipping activity in the X-ray binaries is believed to be caused by the obscuration of $\mathrm{X}$-rays from the compact object by structures located in the outer regions of the disk particularly believed to be impact region of the accretion flow from the binary companion (White \& Swank 1982). Dipping in general leads to the hardening of the source spectrum because of the removal of the soft X-ray photons by photoelectric absorption. The observed properties during the dips viz. duration of the dip, depth of the dip, spectral behavior of the object during the dip etc. show strong variation from dip to dip. The dipping behavior repeats with the orbital period of the binary system. In the dipping low mass Xray binary (LMXB) systems, the hardening of the energy spectrum during the dipping activities is often described by the "progressive covering" or "complex continuum" approach (Church et al. 1997). This approach explains the spectral changes during the dipping intervals by the partial and progressive covering by an opaque neutral absorber. It is found that the dipping activities in the X-ray intensity are very common in LMXBs (Parmar et al. 1999; Narita et al. 2003; Balman 2009 and references therein). However, these activities are rarely seen in the high mass X-ray binary (HMXB) systems.

In case of GX $1+4$, though not an HMXB, intensity dips were seen in the X-ray light curves during which pulsation in soft X-rays were absent (Naik et al. 2005 and references there in). These dips were interpreted as due to the absorption of soft X-ray photons by dense medium. In case of HMXB pulsar GX 301-2, an unusual

\footnotetext{
${ }^{1}$ Astronomy \& Astrophysics Division, Physical Research Laboratory, Ahmedabad - 380009, India.snaik@prl.res.in

2 Raman Research Institute, Sadashivnagar, C. V. Raman Avenue, Bangalore 560080, India
}

intensity dip was observed in 1984 April. A drop in the $\mathrm{X}$-ray continuum intensity (in $7-20 \mathrm{keV}$ band) by an order of magnitude was detected which lasted for about 5 hrs (Leahy et al. 1988). The $6.4 \mathrm{keV}$ iron fluorescent line flux was also reduced to about half with an increase of the equivalent width during the unusual event. The intensity dip observed in the non-eclipsing HMXB pulsar GX 3012 was explained by an obscuration of the neutron star by dense matter which is smaller in size than the scattering region surrounding the neutron star. Dipping activity has been also recognized in 4U $1907+09$ during which the X-ray intensity faded below the detection threshold of the RXTE/PCA. These dips in 4U 1907+09 are interpreted as due to the cessations of the mass accretion by the neutron star (in't Zand et al. 1997). The dips in Vela X-1 are described as the onset of the propeller effect, which inhibits further mass accretion from the binary companion (Kreykenbohm et al. 2008). This are the only known HMXB pulsars showing the presence of intensity dip in the X-ray light curve. Here is this paper, we discuss the presence of several intensity dips in the X-ray light curve of another HMXB pulsar Cen X-3 during its orbit using data from Suzaku observatory.

Cen X-3 was the first binary pulsar to be discovered in X-rays (Giacconi et al. 1971). It is an eclipsing HMXB pulsar with a pulse period of $\sim 4.8 \mathrm{~s}$ and an orbital period of $\sim 2.1$ days ((Schreier et al. 1972). The binary system consists of a neutron star with a mass of $1.21 \pm 0.21 \mathrm{M}_{\odot}$ accompanied by an O 6-8 III supergiant star (V779 Cen) with a mass and radius of $20.5 \pm 0.7 \mathrm{M}_{\odot}$ and $12 \mathrm{R}_{\odot}$, respectively (Hutchings et al. 1979; Rappaport \& Joss 1983; Ash et al. 1999). The distance to the binary system was earlier estimated to be $\sim 8 \mathrm{kpc}$ (Krzeminski 1974). However, using the energy-resolved dust-scattered X-ray halo, Thompson \& Rothschild (2009) estimated the dis- 
tance to Cen X-3 to be $5.7 \pm 1.5 \mathrm{kpc}$. The high luminosity of the X-ray source $\left(\sim 5.0 \times 10^{37} \mathrm{erg} \mathrm{s}^{1}\right.$; Suchy et al. 2008) suggests that the predominant mode of accretion is via a disc, fed by incipient Roche-lobe overflow, although a strong stellar wind does emanate from the supergiant (Nagase et al. 1992; Day \& Stevens 1993). The optical light curve (Tjemkes et al. 1986) supports the presence of an accretion disk, fed by Roche lobe overow. Furthermore, Quasi Periodic Oscillations (QPOs) observed at $\sim 40 \mathrm{mHz}$ (Takeshima et al. 1991; Raichur \& Paul 2008a) strengthen the case for the presence of an accretion disk.

The broad-band, (0.12-100 keV) out-of-eclipse pulsephase-averaged spectrum of Cen X-3 is generally described by an absorbed power-law plus a broad iron emission line at $\sim 6.7 \mathrm{keV}$ along with a high energy cut-off at $\sim 14 \mathrm{keV}$ (Santangelo et al. 1998). A soft excess found in the spectrum below $1 \mathrm{keV}$ has been interpreted as a black-body with $k T \sim 0.1 \mathrm{keV}$ (Burderi et al. 2000) that is now known to be present in many binary X-ray pulsars (Paul et al. 2002; Naik \& Paul 2004a, 2004b and references therein). A cyclotron resonance feature at $\sim 28 \mathrm{keV}$ has also been detected and the corresponding magnetic field strength close to the surface of the neutron star was calculated to be $\mathrm{B} \sim(2.4-3.0) \times 10^{12} \mathrm{G}$ (Santangelo et al. 1998). A number of emission lines are expected in the $\mathrm{X}$-ray spectrum of this source due to its high luminosity $\left(\sim 10^{36-37} \mathrm{erg} \mathrm{s}^{-1}\right)$ and the presence of a strong stellar wind from the companion star (Ebisawa et al. 1996; Wojdowsky et al. 2003; Iaria et al. 2005). Investigation of emission lines, in particular iron lines in 6.4-7.1 keV energy range, at different orbital phases provide significant information on the physical condition of the system. It is understood that the $6.4 \mathrm{keV}$ iron emission line is due to fluorescence of cold material close to the neutron stars (Nagase 1989) where as the $6.7 \mathrm{keV}$ and $6.9 \mathrm{keV}$ lines are considered to be originated in the highly photoionized accretion disk corona (Kallman \& White 1989). ASCA observation of Cen X-3 clearly resolved three iron emission lines at $6.4 \mathrm{keV}, 6.7 \mathrm{keV}$, and $6.9 \mathrm{keV}$ during the eclipse and out-of-eclipse data. The line parameters during the eclipse and out-of-eclipse data confirmed that the $6.4 \mathrm{keV}$ fluorescent line is emitted from the region close to the neutron star where as the highly photoionized plasma that emits 6.7 and $6.9 \mathrm{keV}$ lines is more extended than the size of the companion star (Ebisawa et al. 1996).

In the present work, we have carried out an extensive spectral analysis of the Suzaku observation of the HMXB pulsar Cen X-3 covering the eclipse, out-ofeclipse and the rarely observed dipping activity. For this purpose, we describe the observations, data analysis \& results in the following section. Then in the next section, we discuss the results.

\section{OBSERVATION, ANALYSIS AND RESULTS}

Cen X-3 was observed with the Suzaku satellite from 2008 December 8 06:55:36 to 2008 December 10 05:00:19 using the XISs, HXD/PIN and HXD/GSO detectors. The observation has a total integration time of 97.587 ks. Suzaku, the fifth Japanese X-ray astronomy satellite (Mitsuda et al. 2007), covers $0.2-600 \mathrm{keV}$ energy range with the two sets of instruments, X-ray Imaging Spectrometers (XIS) covering $0.2-12 \mathrm{keV}$ energy range, and the Hard X-ray Detectors (HXD) which covers 10-70 $\mathrm{keV}$ with PIN diodes and $30-600 \mathrm{keV}$ with GSO scintillators. Among the 4 sets of XISs, XIS- 1 is back illuminated (BI) whereas XIS-0, XIS-2, and XIS-3 are front illuminated (FI). The field of view of the XIS is $18^{\prime} \times 18^{\prime}$ in a full window mode with an effective area of $340 \mathrm{~cm}^{2}$ (FI) and $390 \mathrm{~cm}^{2}(\mathrm{BI})$ at $1.5 \mathrm{keV}$. The HXD is a non-imaging instrument that is designed to detect high-energy X-rays. The effective areas of PIN and GSO detectors are $\sim 145$ $\mathrm{cm}^{2}$ at $15 \mathrm{keV}$ and $315 \mathrm{~cm}^{2}$ at $100 \mathrm{keV}$ respectively. For a detailed description of the XIS and HXD detectors, refer to Koyama et al. (2007) and Takahashi et al. (2007). Cen X-3 was observed in HXD nominal mode. As XIS-2 is no more operational, data from other 3 XISs are used in the present analysis.

We used public data (ver-2.2.11.22) from a Suzaku observation of the pulsar in the present work. We used heasoft6.5.1 for our analysis. FTOOLS packages such as PIN - hxdtime, hxdpi, hxdgrade, XIS - xispi, sisclean were applied to the unfiltered event files with standard screening criteria to create cleaned XIS and PIN event files. The effective exposures, after applying the deadtime corrections to the XIS and HXD/PIN data, are found to be $48.452 \mathrm{ks}$ and $79.656 \mathrm{ks}$, respectively. Barycentric correction was then applied to the reprocessed XIS and PIN event files using aebarycen task of FTOOLS. Quick tuned $\left(b g d_{a}\right)$ non X-ray background was used for HXD/PIN. Good time interval (GTI) file was generated for the processed PIN data using above background file. For XISs, source region was selected using rectangular box regions in such a way so that it covers the entire source. The background region for XISs is generated by selecting region far away from the source. Response file, released in July 16 2008, was used for HXD/PIN. Ancillary response file (ARF) and redistribution matrix file (RMF) for XISs were generated using xissimarfgen and xisrmfgen of FTOOLs, respectively.

Light curves were extracted from the processed event files for PIN and XISs using the standard procedures with $4 \mathrm{~s}$ and $100 \mathrm{~s}$ resolutions. Figure 1 shows the XIS-0 and HXD/PIN light curves with $100 \mathrm{~s}$ time resolution. From the figure, it is found that the light curves contain distinctive features of eclipsing, out-of-eclipse phase and dips. This compelled us to do the time resolved spectroscopy of the source in these regions. Source and background spectra were extracted from the processed event data for three XISs and PIN. Dead time correction (using ftool task hxddtcor) was applied to the PIN source spectrum. To determine the start and end of the eclipse, we folded the light curves obtained from the RXTE/ASM dwell data and the Suzaku HXD/PIN event data using the orbital period of 2.08706 d (Raichur \& Paul 2008b). It is found that the eclipse covers $\sim 0.2$ orbital phase range ( $\sim 0.42$ days; Raichur \& Paul 2008b) of the binary system. We figured out the eclipse in the Suzaku observation to be at the beginning and the end of the observation (marked by horizontal lines in both panels of Figure 11). Apart from these two eclipsing parts in the light curve, there are several other low X-ray flux regions which are dips. Light curves at different energy bands were extracted and after appropriate background subtraction are plotted together in Figure 2. From the figure, it can be seen that the dipping feature in the light 


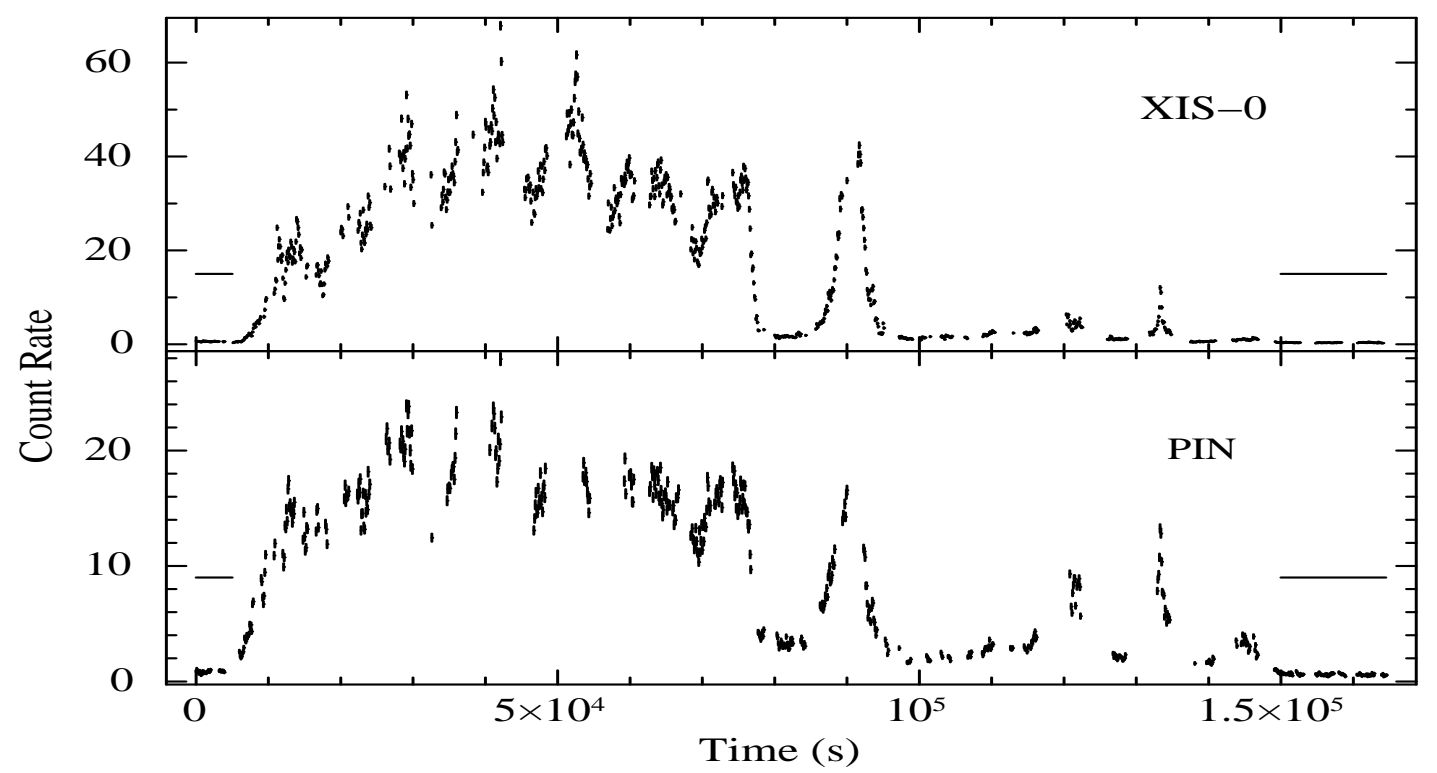

FIG. 1.- Light curves (with $100 \mathrm{~s}$ binning time) obtained from the Suzaku observation of the high mass X-ray binary pulsar Cen X-3. Data from XIS-0 and HXD/PIN detectors are plotted here. The observation was carried out covering nearly entire orbit of the pulsar. The horizontal lines in the beginning and end of the XIS and PIN light curves in both the panels show the duration of eclipse covered during the Suzaku observation.

curve is present up to $\sim 40 \mathrm{keV}$ beyond which the light curve seems to be feature-less.

To understand and compare the properties of the $\mathrm{X}$ ray source during the dips, eclipse and the rest of the regions, the entire light curve was divided into different segments viz. eclipse, eclipse-ingress, eclipse-egress, high count rate region, dip-ingress, dip, dip-egress etc. The light curve showing the various segments is plotted in Figure 3. Each alphabet in the figure represents certain duration for which the spectral analysis was carried out. We extracted XISs and HXD/PIN source spectra for all the segments by applying suitable time filters (as shown in Figure 3) in XSELECT. These spectra contain 2 eclipses, 3 dips, 4 egresses, 5 ingresses, 6 high count regions and 2 bumps with different exposure time and count rates. Dead time corrections were applied to all of the 22 HXD/PIN spectra. After appropriate background subtraction, simultaneous spectral fitting was done using the XIS and PIN spectra with XSPEC V12. All the spectral parameters other than the relative normalization, were tied together for all the detectors. Because an artificial structure is known to exist in the XIS spectra at around the Si edge, we ignored energy bins between $1.75-1.85 \mathrm{keV}$ in the spectral analysis. Apart from the Si edge, large fit residuals due to calibration uncertainties are often observed near the edge structures of the XIS/XRT instrumental responses. Therefore, additional model components for possible fluorescence lines at energies below $\sim 3.5 \mathrm{keV}$ are not considered during the spectral fitting. The relative instrument normalizations of the three XISs and PIN detectors were kept free and the values are found to be in agreement with that at the time of detector calibration.

In order to evaluate the spectra during the eclipses and dips in the light curve in a model-independent manner, we normalized them to that of the high count rate segment (out-of-eclipse and non-dip region). The resulting ratios in XIS and HXD/PIN energy bands are presented in Figure 4. For simplicity, we used only XIS-0 data to obtain the spectral ratio and plotted in the figure. The ratios in XIS energy band indicate the enhancement in the equivalent width of three iron emission lines during the eclipses and dips in the light curve compared to the high count rate segment. Apart from the iron emission lines, the ratios also indicate some changes in the shape of the continuum. The ratios in HXD/PIN energy band (right panels of Figure 4) show that the photons up to $\sim 40 \mathrm{keV}$ are affected by the absorption/obscuration during the dips (bottom two panels) in the light curve. During the eclipse segments, however, the photons in entire energy band are affected in same manner as the ratios (top two panels) are more or less constant.

To quantify the inference from Figure 4, we have done a simultaneous spectral fitting of the XIS and PIN data during the high X-ray count rate region to find a suitable model explaining the spectral features in the source. The broad-band energy spectra (in $0.8-70 \mathrm{keV}$ energy range) were fitted with a model consisting a power-law continuum component modified with edge and high energy cutoff along with the interstellar absorption, and a Gaussian function for the iron fluorescence line at $6.4 \mathrm{keV}$. Presence of line like features in the residuals at $6.7 \mathrm{keV}$ and $6.9 \mathrm{keV}$ allowed us to add two more Gaussian functions with the spectral model. Initially we ignored data below $4 \mathrm{keV}$. A cyclotron resonance feature at $\sim 30 \mathrm{keV}$ was also found in the spectral fitting. However, as the cyclotron features in this pulsar will be presented elsewhere, we concentrated on the variation of other spectral parameters at different X-ray intensity phases over the binary orbit. Though the above model, with the cyclotron resonance feature, fitted well to the high count rate spectra, there were inconsistency in the value of other spectral parameters during several other segments where the values of high energy cut-off and folding energy are found to be $<4 \mathrm{keV}$ i.e. beyond the fitted energy band) with power-law photon-index significantly different to that during the high count rate segments. Therefore, the above model was not suitable for the spectral 


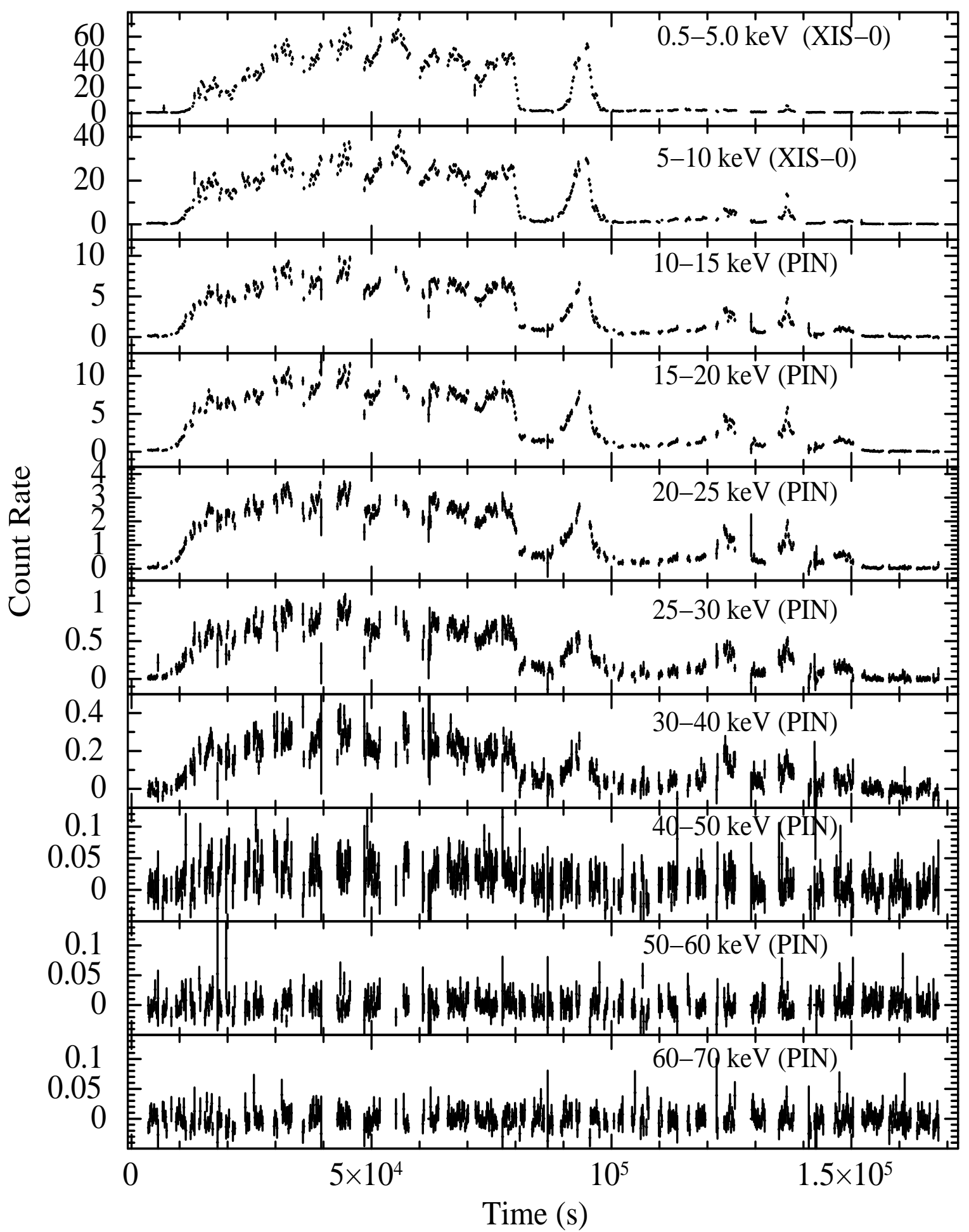

FIG. 2.- Light curve (with $200 \mathrm{~s}$ binning time) obtained from the Suzaku observation of the high mass X-ray binary pulsar Cen X-3. Data from XIS-0 and HXD/PIN detectors are plotted here. The observation was carried out covering nearly entire orbit of the pulsar. 


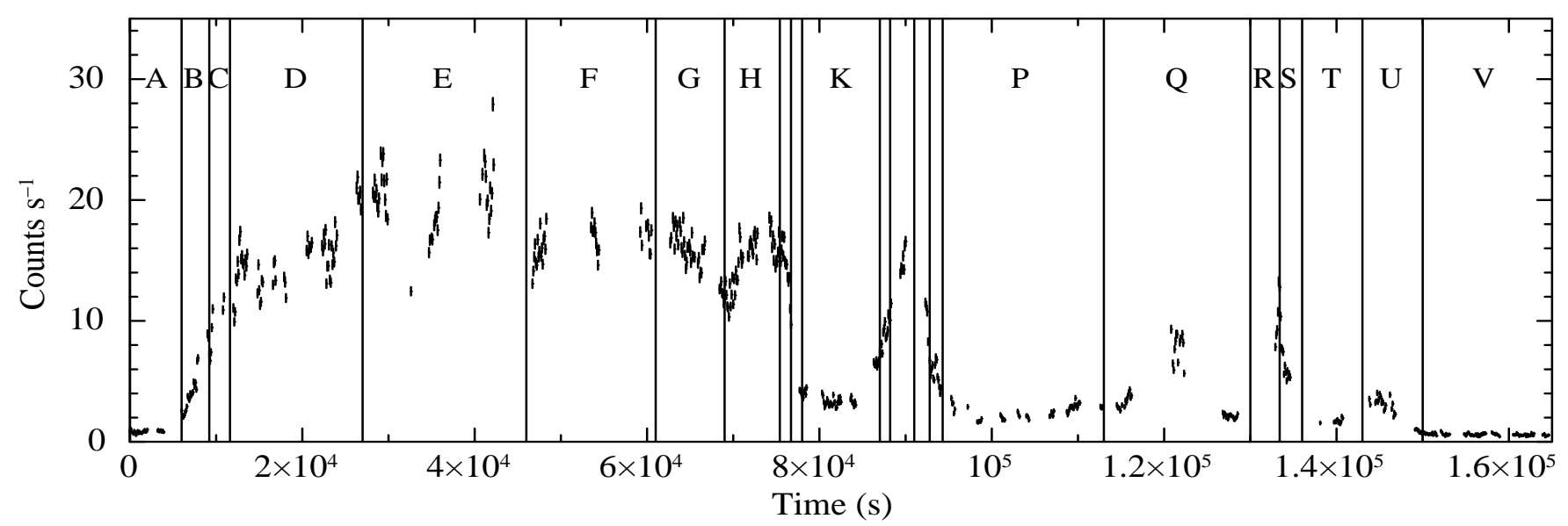

FIG. 3.- HXD/PIN light curve (with $100 \mathrm{~s}$ binning time) obtained from the Suzaku observation of the high mass X-ray binary pulsar Cen X-3. The entire light curve is divided into various segments (marked with alphabets) of different duration based on the presence of eclipse, dips, and high count rate phase. The smaller duration regions are not marked with the alphabets in the figure. However, the nomenclature goes with sequence and the corresponding spectral parameters are given in Table-1.
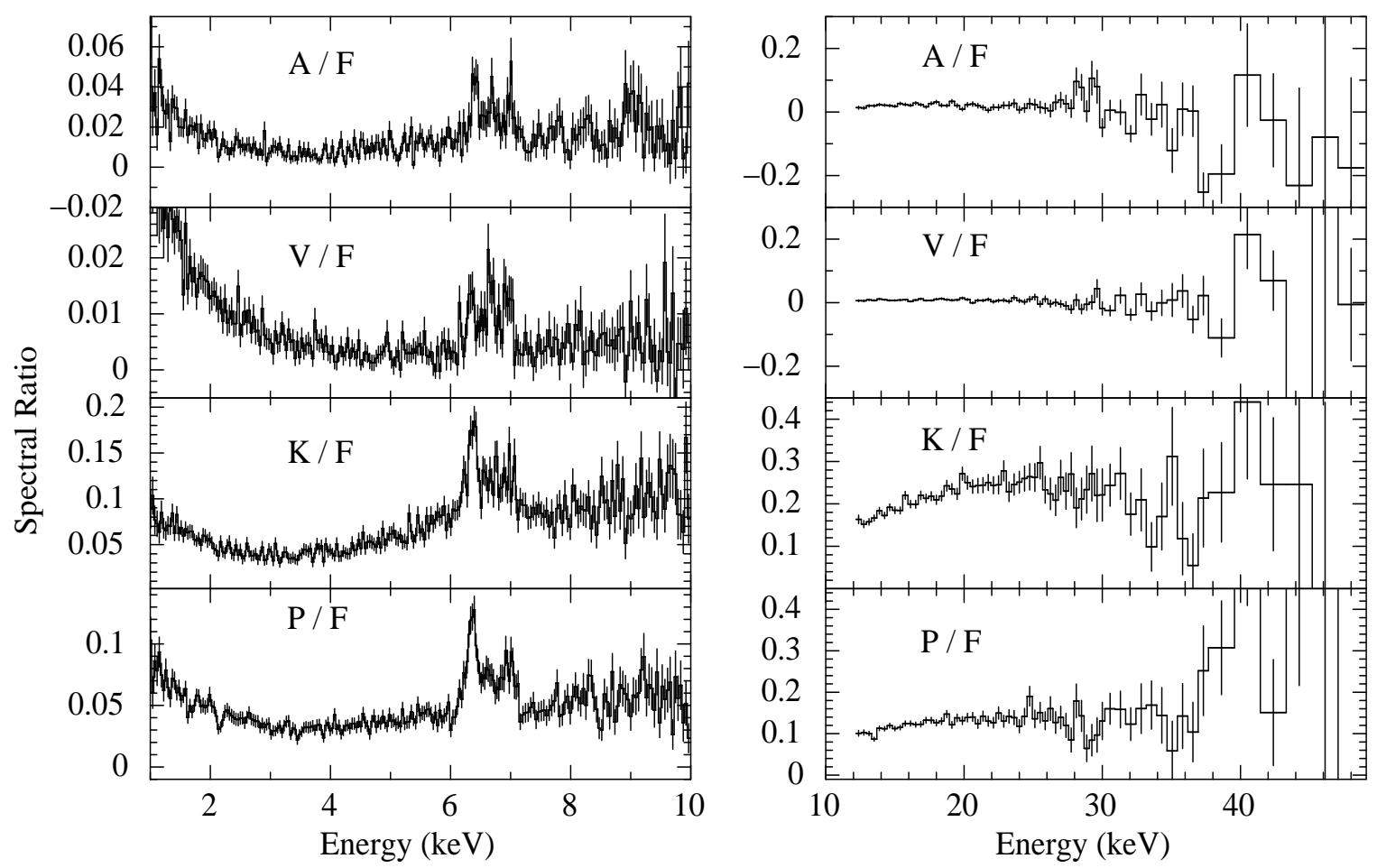

FIG. 4. - Ratios of the background-subtracted spectra of Cen X-3 during the eclipses (marked as 'A' and 'V') and dips (marked as 'K' and ' $\mathrm{P}$ ') to that of the high X-ray intensity segment (marked as ' $F$ ') in the light curve (as shown in Figure 3). The panels in left side are obtained from the XIS-0 data where as in right side, obtained from the HXD/PIN data. 
fitting to the spectra of all the segments.

We then tried to explore other continuum models to get a better fit to the broad band spectrum of Cen X-3. In the process, we found that a partially covering high energy cutoff power-law continuum model (as in other cases; Naik et al. (2011) and references therein), along with three Gaussian functions, Galactic absorption, and the cyclotron resonance feature, fits the pulsar spectrum with acceptable parameters for the spectra of all the segments. The partial covering model consists of two powerlaw continua with a common photon index but with different absorbing hydrogen column densities. Once we found the suitable continuum model, we extended the fitting to low energies $(\sim 0.8 \mathrm{keV})$ and estimated the spectral parameters for all the segments. The time-resolved count rate spectra, along with the best-fit model components, of the pulsar Cen X-3 are shown in Figure 5 for eclipse (A and V), eclipse-egress (B), high count rate segments ( $\mathrm{D}$ and $\mathrm{F})$, dip $(\mathrm{K}, \mathrm{P}$ and $\mathrm{T})$, dip-ingress $(\mathrm{O})$ segments (as shown in Figure 3). In the figure, the iron emission lines are found to be most prominent during the dips and eclipse segments.

The best-fit parameters obtained from the simultaneous spectral fitting to the XIS and PIN data for all the segments (as given in Figure 3) of the Suzaku observation of Cen X-3 are given in Table 1] The values of the additional absorption column density $\left(\mathrm{N}_{\mathrm{H} 2}\right)$ are found to vary in a wide range starting from $\sim 10^{22}$ atoms $\mathrm{cm}^{-2}$ to $132 \times 10^{22}$ atoms $\mathrm{cm}^{-2}$. The significantly high values of $\mathrm{N}_{H 2}$ along with the corresponding covering fractions during dips and the eclipse segments in the light curve are understood to be because of the obscuration/absorption of the X-ray photons from the pulsar by dense matter along the line of sight. Apart from the significantly high value of $\mathrm{N}_{\mathrm{H} 2}$ and covering fractions at the dips and eclipse segments, the iron emission line parameters are also observed to be very different from those during the high count rate segments. The change in values of $\mathrm{N}_{H_{2}}$, covering fraction and the power-law photon index over the orbital phase of the pulsar during the Suzaku observation are shown in Figure 6. In the figure, the mideclipse time (derived from the orbital parameters given in Table 1 of Paul et al. 2005) is considered as phase zero. From Table 1 and Figure [6] it can be seen that the values of $\mathrm{N}_{H 2}$ and covering fraction for the spectral segment ' $\mathrm{O}$ ' are different from that of during the dips/eclipse segments or high count rate segments. The value of $\mathrm{N}_{H 2}$ is high and found to be comparable to that in the next segment, which is a dip. But the covering fraction is only about $\sim 0.5$. This is consistent with the scenario of progressive covering.

The dependence of the three iron $\mathrm{K}_{\alpha}$ emission line intensities and equivalent widths on the X-ray intensity at different segments of the orbit is shown in Figure 7. It is found that the line intensities are minimum during the eclipse segments. During the dips, though the lines are comparatively more intense than during the eclipse segments, the line flux is significantly low compared to the high count rate segments. The equivalent width of the $6.4 \mathrm{keV}$ line during dips is found to be comparable to that during the eclipse segments which is $\sim 400 \mathrm{eV}$. However, the equivalent width of the 6.7 and $6.97 \mathrm{keV}$ lines during dips were significantly low compared to that dur- ing the eclipse segments. These results suggest that the iron emission line parameters during the dip evolve due to a progressive covering. Compared to the $6.4 \mathrm{keV}$ line, the 6.7 and $6.97 \mathrm{keV}$ lines evolve slightly differently as they originate further away from the neutron star. This indicates that the dipping activity during the Suzaku observation of Cen X-3 is possibly because of the presence of structures in the outer region of the accretion disk, as seen in low mass X-ray binary systems.

Table 1 and Figure [6] show that the source spectrum during the eclipse segments ('A' and ' $V$ ' in Figure 3) is steep compared to the rest of the binary phases; the power-law photon index is as high as 3, where as during the other segments, it is $\sim 1$. We attempted to fit the eclipse spectrum using a high energy cutoff powerlaw model with interstellar absorption and three Gaussian functions for the iron emission lines. The best-fit parameters for the simultaneous spectral fitting to the eclipse data using this model are given in the last line of Table 1. The high value of power-law index during the eclipse compared to the rest of the orbital phases is seen earlier observations of Cen X-3 (Thompson \& Rothschild 2009 and references therein). Similar changes in the spectral slope during the eclipse and rest of the binary phases are also seen in $4 \mathrm{U}$ 1538-52 (Robba et al. 2001). The observed spectral steepness during the eclipse of these objects are attributed to the interstellar dust scattering. Numerous studies of the eclipse and out-ofeclipse spectra of these type of objects found that the dust scattered component has a spectrum that is steeper by a factor proportional to $E^{-2}$ that affects the eclipse spectrum most (Nagase et al. 2001; Robba et al. 2001; Clark 2004; Thompson \& Rothschild 2009).

\section{DISCUSSION}

The high mass X-ray binary pulsar Cen X-3, though, has been observed at many different epochs with various observatories, Suzaku observation of the pulsar in 2008 December 8-10 is one of the longest imaging spectroscopic observation. This observation covers almost the entire orbital period of the binary system. Significant flux variation over the orbit has been seen in the $\mathrm{X}$-ray light curve of the pulsar. Presence of dips in the light curve which is common in the LMXBs and rare in HMXBs, is the key feature of the observation. A detailed orbital phase resolved spectroscopy of the pulsar in wide $\mathrm{X}$-ray band is, therefore, very important to understand the properties of the binary system.

Selection of an appropriate continuum model is important to investigate the presence of several features such as the soft excess represented by a blackbody component, emission lines, cyclotron absorption features etc. in the X-ray binary pulsar spectrum. The phenomenological model which has been commonly used to describe the continuum spectra of X-ray binary pulsars is a combination of a power law of photon index $\sim 1$, a black-body component with a temperature of few hundreds of $\mathrm{eV}$ and a high energy cut-off above $10 \mathrm{keV}$. Over and above, there are emission lines from ions and broad absorption lines due to cyclotron resonances (Orlandini 2006). In case of a few other X-ray binary pulsars, it has been reported that the absorption has two different components (Endo et al. 2000; Mukherjee \& Paul 2004; Naik et al. 2011 and references therein). In this model, one absorption com- 

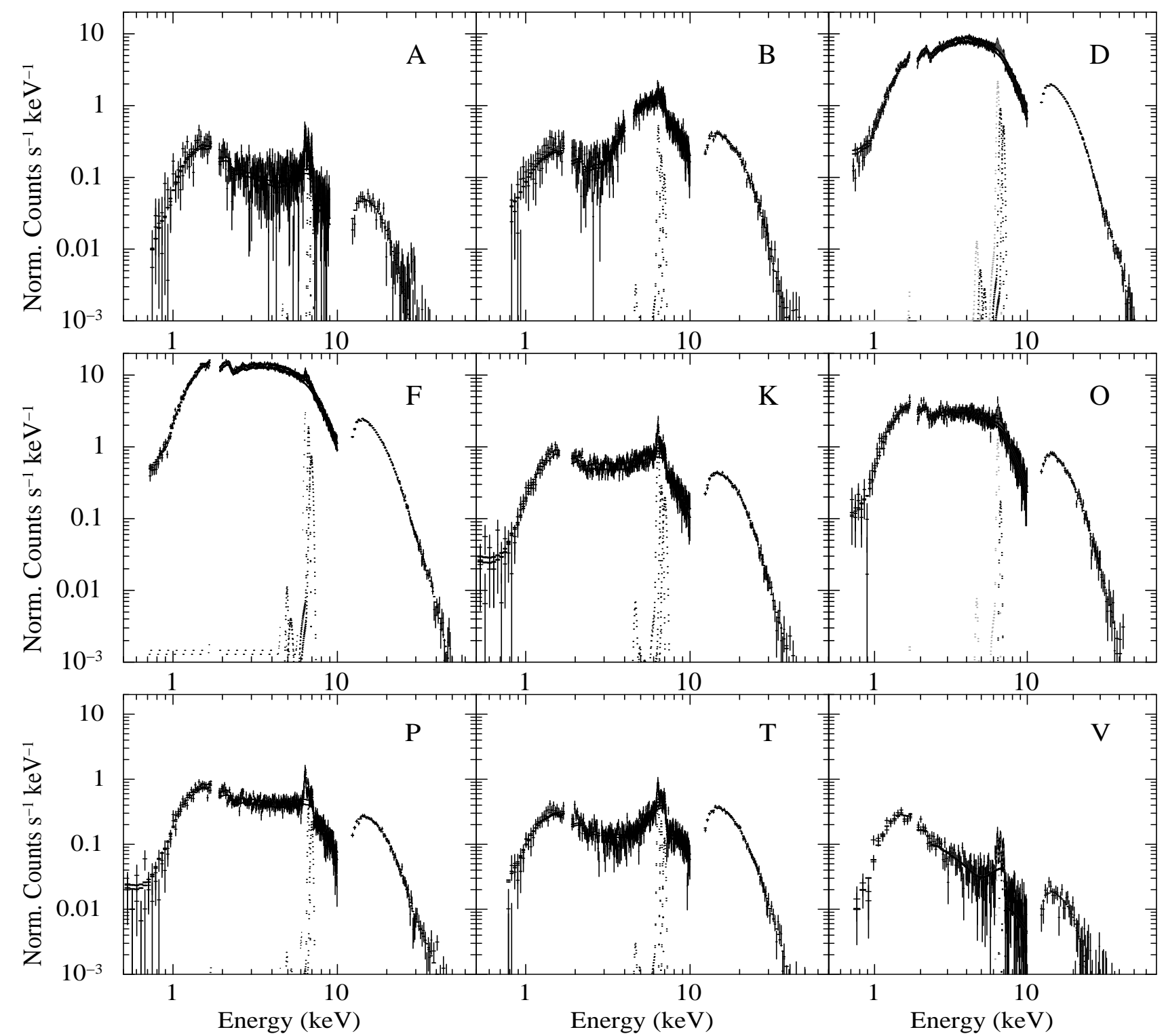

FIG. 5. - Time resolved energy spectra of Cen X-3 obtained with the XIS-0, XIS-3, and PIN detectors of the Suzaku observation, along with the best-fit model comprising a partially absorbed high energy cutoff power-law continuum model, three iron line emissions at 6.4 $\mathrm{keV}, 6.7 \mathrm{keV}$, and $6.97 \mathrm{keV}$ and cyclotron resonance features at $\sim 30 \mathrm{keV}$. The alphabets in the top right corner of each spectra represent the region from which data were used for spectral fitting (as shown in Figure 3). The variation in the continuum level and also the iron emission lines are seen in the spectra.

ponent absorbs the entire spectrum where as the other component absorbs the spectrum partially. This model is known as partial covering absorption model. Though the power law with high energy cutoff model explains the pulse phase averaged pulsar spectra well, it runs into problem while fitting the spectra during the dip phases of pulsars showing dips or dip-like features in the pulse profiles. However, the partial absorption model fits very well to all the pulse phase resolved spectra of these pulsars (Her X-1 - Endo et al. 2000, GRO J1008-57 - Naik et al. 2011; 1 A 1118-616 - Devasia et al. 2011a, Maitra et al. 2011; GX 304-1 - Devasia et al. 2011b). This model has also been used to describe the orbital phase resolved spectroscopy in some of the high mass X-ray binary systems such as Cen X-3 (present work), GX 301-2 (Mukherjee \& Paul 2004; Naik et al. 2009) to explain the variation of the absorption column density over the binary orbit.

In the partial covering model, $N_{\mathrm{H} 2}$ is interpreted as the column density of the material that is local to the X-ray source, while $N_{\mathrm{H} 1}$ accounts for the rest of the material along with the Galactic absorption. The covering fraction is defined as Norm $2 /($ Norm $1+$ Norm 2$)$ where Norm 1 and Norm 2 are normalizations of the two power laws respectively. It is seen from Table-1 that the $N_{\mathrm{H} 2}$ and covering fraction remain substantially high (> $50 \times 10^{22}$ units and $>80 \%$, respectively) during the dips and the eclipse regions in the light curve. This means that there is dense and clumpy material present at certain orbital phases of the Cen X-3 binary system causing dips in the light curve. A study of iron emission line parameters during eclipse-ingress, eclipse and eclipse-egress with the $A S C A$ noted that the $6.4 \mathrm{keV}$ fluorescent line is emitted from a region close to the neutron star where 
TABLE 1

Best-fit parameters (with $1 \sigma$ errors) of the phase-averaged spectra for Cen X-3 during the Suzaku observation.

\begin{tabular}{|c|c|c|c|c|c|c|c|c|c|c|}
\hline \multirow{2}{*}{$\begin{array}{l}\text { Spec. } \\
\text { Reg. }\end{array}$} & \multirow[t]{2}{*}{$\mathrm{N}_{H 1}$} & \multirow[t]{2}{*}{$\mathrm{N}_{H 2}$} & \multirow{2}{*}{$\begin{array}{l}\text { Cov. } \\
\text { Fraction }\end{array}$} & \multirow{2}{*}{$\begin{array}{l}\text { Photon } \\
\text { Index }\end{array}$} & \multicolumn{2}{|c|}{$6.4 \mathrm{KeV}$ line } & \multicolumn{2}{|c|}{$6.7 \mathrm{KeV}$ line } & \multicolumn{2}{|c|}{$6.97 \mathrm{KeV}$ line } \\
\hline & & & & & Flux $^{a}$ & $\mathrm{Eqw}^{b}$ & Flux $^{a}$ & $\mathrm{Eqw}^{b}$ & Flux $^{a}$ & \\
\hline $\mathrm{A}$ & $1.06 \pm 0.03$ & $.1 \pm 3.0$ & $0.94 \pm 0.01$ & $2.03 \pm 0.02$ & $0.30 \pm 0.03$ & $0.43 \pm 0.05$ & $0.20 \pm 0.02$ & $0.26 \pm 0.03$ & $0.13 \pm 0.03$ & $\overline{0.20 \pm 0.04}$ \\
\hline B & $50 \pm 0.04$ & $4 \pm 0.6$ & .43 & 05 & $50 \pm 0.11$ & .02 & $.24 \pm 0.11$ & $03 \pm 0.02$ & & \\
\hline $\mathrm{C}$ & $33 \pm 0.03$ & 0.2 & 0.01 & $=0.01$ & $.80 \pm 0.16$ & $.08 \pm 0.01$ & $.43 \pm 0.17$ & $0.02 \pm 0.01$ & & \\
\hline $\mathrm{D}$ & $3 \pm 0.01$ & 0.1 & & 01 & $55 \pm 0.01$ & 01 & $1.29 \pm 0.09$ & $0.04 \pm 0.01$ & $.90 \pm 0.10$ & 0.03 \\
\hline $\mathrm{E}$ & & & & 01 & & & $69 \pm 0.10$ & & & \\
\hline $\mathrm{F}$ & & & & 01 & $.78 \pm 0.18$ & & $92 \pm 0.13$ & & .13 & 0.01 \\
\hline G & & & & 1 & & & .13 & & & .01 \\
\hline $\mathrm{H}$ & & & & 1 & \pm 0.14 & & .19 & & .20 & .01 \\
\hline I & & & & 1 & & & .29 & & .30 & .01 \\
\hline $\mathrm{J}$ & .02 & 4 & & 1 & $=0.18$ & & \pm 0.13 & & \pm 0.21 & .02 \\
\hline $\mathrm{K}$ & & & & 1 & & & .06 & & \pm 0.06 & 0.01 \\
\hline $\mathrm{L}$ & & & & 1 & .20 & 0.2 & \pm 0.19 & & \pm 0.22 & 0.01 \\
\hline M & & & & 1 & & & \pm 0.29 & & \pm 0.32 & \\
\hline $\mathrm{N}$ & 0.01 & 0.2 & & 01 & $2.89 \pm 0.26$ & 0.0 & $1.28 \pm 0.33$ & 0.01 & $3 \pm 0.52$ & 0.01 \\
\hline $\mathrm{O}$ & $1.29 \exists$ & $92.9 \pm 5.4$ & .01 & $1.01 \pm 0.01$ & $1.30 \pm 0.15$ & $0.11 \pm 0.01$ & $0.21 \pm 0.11$ & $0.02 \pm 0.01$ & $0.01_{-0.01}^{+0.10}$ & $0.01 \pm 0.01$ \\
\hline $\mathrm{P}$ & & $9.2 \pm 1.9$ & & & & & 0.03 & 0.01 & $0.35 \pm 0.04$ & 0.02 \\
\hline Q & $80 \pm 0.02$ & $6.3 \pm 0.7$ & & $0.92 \pm 0.01$ & $1.23 \pm 0.05$ & $0.21 \pm$ & $0.57 \pm 0.04$ & $0.09 \pm 0.01$ & $0.61 \pm 0.04$ & $0.10 \pm 0.01$ \\
\hline $\mathrm{S}$ & $85 \pm 0.04$ & $33.9 \pm 0.8$ & & $0.58 \pm 0.01$ & $1.61 \pm 0.12$ & $0.18 \pm 0.01$ & $1.10 \pm 0.20$ & $0.12 \pm 0.02$ & $1.28 \pm 0.16$ & $0.14 \pm 0.02$ \\
\hline $\mathrm{T}$ & $65 \pm 0.02$ & $85.0 \pm 1.1$ & $96 \pm 0.26$ & $1.31 \pm 0.01$ & $0.51 \pm 0.03$ & $0.24 \pm 0.02$ & $0.24 \pm 0.04$ & $0.10 \pm 0.02$ & $0.20 \pm 0.04$ & $0.09 \pm 0.02$ \\
\hline U & $66 \pm 0.04$ & $84.9 \pm 1.9$ & $=0.45$ & $0.83 \pm 0.02$ & $0.62 \pm 0.04$ & $0.34 \pm 0.02$ & $0.28 \pm 0.06$ & $0.13 \pm 0.03$ & $0.27 \pm 0.05$ & $0.13 \pm 0.03$ \\
\hline V & $1.34 \pm 0.04$ & $132.2 \pm 4.5$ & $0.97 \pm 0.64$ & $2.89 \pm 0.03$ & $0.10 \pm 0.02$ & $0.39 \pm 0.06$ & $0.10 \pm 0.02$ & $0.35 \pm 0.06$ & $0.06 \pm 0.01$ & $0.26 \pm 0.05$ \\
\hline$\overline{\mathrm{V} *}$ & $1.05 \pm 0.05$ & - & - & $2.38 \pm 0.07$ & $0.14 \pm 0.01$ & $0.46 \pm 0.02$ & $0.14 \pm 0.01$ & $0.32 \pm 0.01$ & $0.12 \pm 0.02$ & $0.41 \pm 0.02$ \\
\hline
\end{tabular}

$\mathrm{N}_{H 1}=$ Equivalent hydrogen column density (in $10^{22}$ units), $\mathrm{N}_{H 2}=$ Additional hydrogen column density (in $10^{22}$ units), ${ }^{a}:$ in $10^{-11}$ ergs $\mathrm{cm}^{-2} \mathrm{~s}^{-1}$ unit, ${ }^{b}$ : Equivalent width (in $\mathrm{keV}$ ).

* : Best-fit parameters obtained by fitting the eclipse spectrum (segment 'V') by using a high energy cut-off power law model with interstellar absorption and three Gaussian functions for the three iron emission lines.

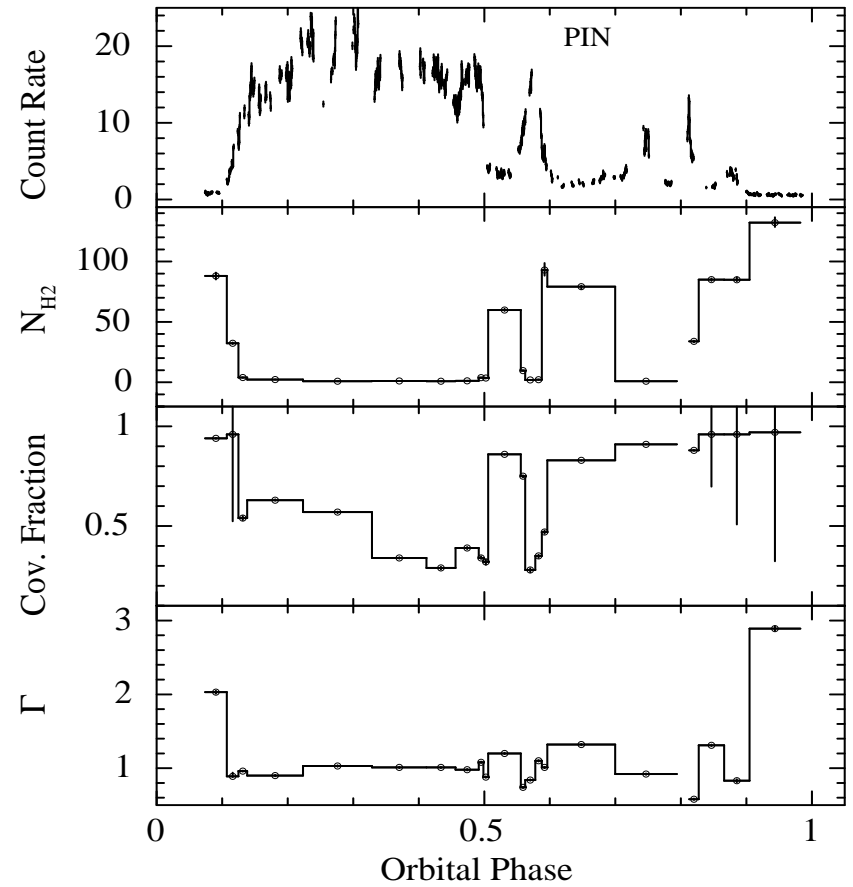

FIG. 6.- The top panel of the figure shows the HXD/PIN light curve (with $100 \mathrm{~s}$ resolution) of the Suzaku observation of Cen X3 . The mid-eclipse time is considered as orbital phase zero. The values of $\mathrm{N}_{H_{2}}$ (in $10^{22}$ units), covering fraction, and power-law photon index obtained from the time resolved spectroscopy of the Suzaku observation are shown in second panel, third panel, and bottom panel, respectively. The errors shown in the figure are estimated for $1 \sigma$ confidence level. as the $6.7 \mathrm{keV}$ and $6.97 \mathrm{keV}$ line emission region is much larger and more extended than the size of the companion star (Ebisawa et al. 1996). This information can be used to compare the iron line parameters during the dips and eclipse segments of the Suzaku observation of the pulsar. It is found that the equivalent widths of the three iron emission lines are significantly larger during the dips and eclipse segments in the light curve (Figure 7). The flux of these lines are also considerably low during these orbital phases compared to that of the high count rate phases in the light curve. The high equivalent width and low flux of iron emission lines at dips and eclipse segments also suggest the presence of significant absorption and reprocessing during corresponding orbital phases of the binary system. This feature is similar to that seen in Her X-1 and LMC X-4 (Naik \& Paul 2003), two other sources that show strong superorbital X-ray intensity variations understood to be due to varying degree of obscuration by the precessing accretion disk.

During the ASCA observation of Cen X-3, unlike the present work, the equivalent width of $6.4 \mathrm{keV}$ line hardly varied during the eclipse-ingress, eclipse, and eclipseegress (Ebisawa et al. 1996). In fact, this is in contrast with the similar HMXBs Vela X-1 and GX 301-2. During the eclipse, in Vela $\mathrm{X}-1$, the $6.4 \mathrm{keV}$ iron line equivalent width increases significantly than that during the outof-eclipse (Nagase et al. 1994 and references there in). Though GX 301-2 does not show binary eclipse, a significant increase in the $6.4 \mathrm{keV}$ iron emission line equivalent width was observed during an intensity dip that is thought to be due to the occultaion of the neutron star by dense matter (Leahy et al. 1988). During the intensity dips in Cen X-3 (present work), it is found that the iron 

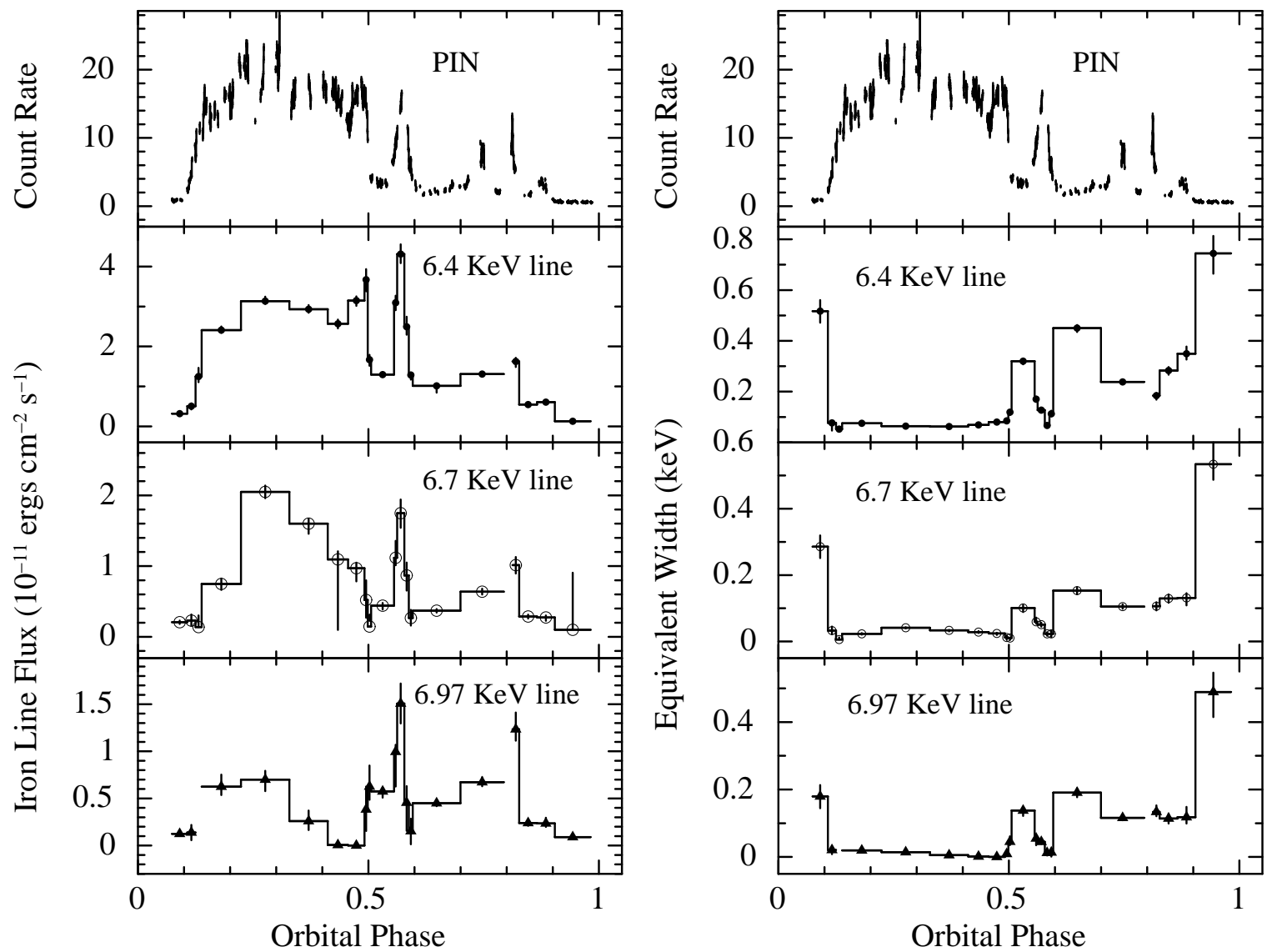

FIG. 7.- Iron emission line parameters obtained from the time resolved spectroscopy of Suzaku observation of Cen X-3. The errors shown in the figure are estimated for $1 \sigma$ confidence level. The top panels show HXD/PIN light curves with $100 \mathrm{~s}$ resolution. The bottom three panels in left side show the change in estimated line flux in $10^{-11} \mathrm{ergs}_{\mathrm{cm}}^{-2} \mathrm{~s}^{-1}$ units where as the bottom three panels in right side show the variation in the equivalent widths for three iron emission lines during the entire observation.

emission line intensity dropped by a factor of 2 to 3 with significant increase in the line equivalent widths. However, the drop in continuum flux is found to be by an order of magnitude or more during the dips (Figure 7 ). The decrease in the continuum flux during the dips suggest that the direct power law component in the partial covering model is almost vanished or completely absorbed where as the second power law component contributes to the X-ray flux observed during the dips. Therefore, the presence of dips in the X-ray light curve of Cen X-3 can be explained by the eclipse/obscuration of the neutron star by dense matter which is smaller in size compared to the scattering region surrounding the neutron star, as seen in GX 301-2.

\section{ACKNOWLEDGMENTS}

The research work at Physical Research Laboratory is funded by the Department of Space, Government of India. We express our thanks to the anonymous referee for his/her valuable comments which improved our paper. The authors would like to thank all the members of the Suzaku for their contributions in the instrument preparation, spacecraft operation, software development, and in-orbit instrumental calibration. This research has made use of data obtained through HEASARC Online Service, provided by the NASA/GSFC, in support of NASA High Energy Astrophysics Programs.

\section{REFERENCES}

Ash, T. D. C., Reynolds, A. P., Roche, P., Norton, A. J., Still, M. D., \& Morales-Rueda, L. 1999, MNRAS, 307, 357

Balman, S. 2009, AJ, 138, 50

Burderi, L., Di Salvo, T., Robba, N. R., et al. 2000, ApJ, 530, 429

Church, M. J., Dotani, T., Balucinska-Church, M., Mitsuda, K., Takahashi, T., Inoue, H., Yoshida, K. 1997, ApJ, 491, 388

Clark, G. W. 2004, ApJ, 610, 956

Day, C. S. R. \& Stevens, I. R. 1993, ApJ, 403, 322

Devasia, J., James, M., Paul, B., Indulekha, K. 2011a, MNRAS (Accepted)

Devasia, J., James, M., Paul, B., Indulekha, K. 2011b, MNRAS (Submitted)

Ebisawa, K., Day, C. S. R., Kallman, T. R., et al. 1996, PASJ, 48, 425

Endo, T., Nagase, F., Mihara, T. 2000, PASJ, 52, 223
Giacconi, R., Gursky, H., Kellogg, E., Schreier, E., \&

Tananbaum, H. et al. 1971, ApJ, 167, L67

Hutchings, J. B., Cowley, A. P., Crampton, D. et al. 1979, ApJ, 229,1079

Iaria, R., Di Salvo, T., Robba, N. R., et al. 2005, ApJ, 634, L161 in't Zand, J. J. M., Strohmayer, T. E., \& Baykal, A. 1997, ApJ, 479, L47

Kallman, T. R. \& White, N. E. 1989, ApJ, 341, 955

Kreykenbohm, I., Wilms, J., Kretschmar, P., et al. 2008, A\&A, 492,511

Krzeminski, W., 1974, ApJ, 192, L135

Koyama, K., et al. 2007, PASJ, 59, 23

Leahy, D. A., Nakajo, M., Matsuoka, M., Kawai, N., Koyama, K., Makino, F. 1988, PASJ, 40, 197

Maitra, C. et al. 2011, MNRAS (in preparation) 
Mitsuda, K., et al. 2007, PASJ, 59, 1

Mukherjee, U. \& Paul, B. 2004, A\&A, 427, 567

Nagase, F., Dotani, T., Endo, T., et al. 2001, AIP Conf. Proc., 599,794

Nagase, F., Zylstra, G., Sonobe, T., Kotani, T., Inoue, H., Woo, J. 1994, ApJ, 436, L1

Nagase, F. et al. 1992, ApJ, 396, 147

Nagase, F. 1989, PASJ, 41, 1

Naik, S., Paul, B., Kachhara, C., Vadawale, S. V. 2011, MNRAS, 413,241

Naik, S., Mukherjee, U., Paul, B., Choi, C. S. 2009, Adv. Space Res., 43, 900

Naik, S., Paul, B., \& Callanan, P. J. 2005, ApJ, 618, 866

Naik, S. \& Paul, B. 2004a, ApJ, 600, 351

Naik, S. \& Paul, B. 2004b, A\&A, 418, 655

Naik, S. \& Paul, B. 2003, A\&A, 401, 265

Narita, T., Grindlay, J. E., Bloser, P. F., \& Chou, Y. 2003, ApJ, 593, 1007

Orlandini, M. 2006, Adv. Space Res., 38, 2742

Parmar, A. N., et al. 1999, A\&A, 351, 225

Paul, B., Nagase, F., Endo, T., Dotani, T., Yokogawa, J., Nishiuchi, M. 2002, ApJ, 579, 411

Paul, B., Raichur, H. \& Mukherjee, U. 2005, A\&A, 442, L15
Raichur, H. \& Paul, B. 2008a, ApJ, 685, 1109

Raichur, H. \& Paul, B. 2008b, MNRAS, 387, 439

Rappaport, S. A. \& Joss, P. C. 1983, Accretion Driven Stellar X-ray Sources, ed. W. H. G. Lewin \& E. P. J. van den Heuvel (Cambridge: Cambridge Univ. Press)

Robba, N. R., Burderi, L., Di Salvo, T., Iaria, R., Cusumano, G. 2001, ApJ, 562, 950

Santangelo, A., del Sordo, S., Segreto, A., et al. 1998, A\&A, 340, L55

Schreier, E., Levinson, R., Gursky, H., Kellogg, E., Tananbaum, H., \& Giacconi, R. 1972, ApJ, 172, L79

Suchy, S. et al. 2008, ApJ, 675, 1487

Takahashi, T., et al. 2007, PASJ, 59S, 35

Takeshima, T., Dotani, T., Mitsuda, K., \& Nagase, F., 1991, PASJ, 43L, 43

Thompson, T. W. J. \& Rothschild, R. E. 2009, ApJ, 691, 1744

Tjemkes, S. A., van Paradijs, J., \& Zuiderwijk, E. J., 1986, A\&A, 154,77

White, N. E. \& Swank, J. H. 1982, ApJ, 253, L61

Wojdowski, P. S., Liedahl, D. A., Sako, M., et al. 2003, ApJ, 582, 959 